instruments. Simon (1995) Conventional InformationBased Policies and Management Operations Management Systems are used by management to measure and modify patterns in management operations. Resource management and information management have such policies and routines. Merchant and Ottley (2007), the main intent of the Management Control System, has been identified as information management for planning, evaluation and decision making in company management

Strategic planning applies some items or business portions at any time. Strategies are typically inclusive and broad-based rather than exclusive. For example, the low cost strategy specifies separators and contains the company's entirely different actions. It is rare and necessary due to its natural strategy. Any serious change in an external environment may require reorganization or hard work unless a change in organization's strategy is needed. Thus strategies are associated with the external environment's environment and external environment. Information used to make a decision is not approximate and current data. Strategy is limited to the best management. Generally maintaining the management, instruction and instructions for lower maintenance.

Management control systems are always effective, in design or in terms of implementation. These systems only increase the likelihood of achieving institutional goals of efficiency, efficiency, accuracy of financial statements and compliance. Management controls should be merged or built into the company's programs. These built-in control systems influence company's ability to achieve its goals and also help improve the quality of its business activities. The five components of management control - the control environment, risk assessment, control operations, information and the communication and control system. The policies and procedures used in the organization to provide reasonable assurance that the directions and instructions given by management operations are properly followed.

Business activities, organizational goals, complexity in business operations, people involved in the implementation of these operations and controlling activities on company structure and culture are different. Organizing meetings helps in decisionmaking and reduction of the time taken for the decision-making process. Four different sets of meetings that are used for different purposes: daily check-in, weekly strategic, monthly, strategic and quarterly off-site review. Information systems are not effective without proper communication between different levels of operating system. Information transmission is not just a matter of sending information, but also to work coordination and responsibilities. Two types of communications internal conversations and external dialogues - occur in any organization.

\section{Management Control}

Indirect activities will come under the control of management. Indirect activities include personnel operations such as human resources and marketing. However, line controls are also under management control. Also, management control is a long and short term combination. The strategy of outstanding ownership is divided into functional budgets and notified to operational groups. This is the duty of management control. Hence the maintenance control line and staff functions. Management control is also a two-way process, where communication from operational management to operational teams and vice versa takes place. Management control has also been expanded by expanding all levels of management. Adele, Bradbent and Berry (1995) consider management control as a function that integrates management control and strategic planning. The management control scope is not limited, since it is not limited to reporting interactions and performance management of instructional instructions for lower management. Management controls between upper and lower levels will be because it will report low levels of strategies and operational capabilities for the best management. The strategy has a long-term vision and has little focus on activities. Management control is a combination of both planning and control.

Regulatory activities are monitored on a continuous basis or separately. Continuous supervision helps the organization by providing feedback on whether the auxiliary parts of the company are efficient or ineffective. A special assessment of operations helps to fully understand the effectiveness of the control system and is continuous monitoring processes. The most important factor in implementing regulatory systems is the proper procedures for organizations to identify, communicate, follow, and correct (if any) organizations. Management control controls the number of individuals internal and external. Each of them plays a different role and has different responsibilities towards the effective implementation of the management control system. The company's 
internal management, the board of directors, internal auditors and many employees; External auditors, regulatory agencies, customers, distributors and financial analysts are related to the company's external entities.

Control is a process implemented by the people, and related processes should be practiced mechanically rather than thought. Another important requirement for the success of the administrative control systems administration in an organization is the execution. Problems affecting the management control process that performs the management control system or the exceptional consequences are in effect. Some problems that may hinder management control process: lack of proper organizational structure, management style, well defined hierarchy, etc. Proper person job and person-reward fit; Deficiencies of training and development of employees; Conspiracy between the controller and the control person; Illegal use of management authority; Lack of proper conversation.

\section{Fundamentals of Management Control}

Management terminology, 'control', refers to the function of establishing traditional performance traditionally, assessing actual performance against these standards and implement appropriate actions to achieve company objectives. In complex and dynamic modern business environment, management control systems are used to meet efficiency, efficiency and adaptive learning requirements. It is widely anxious to implement management control targets and implement strategies. "Management and other groups can initiate a behavioral approach and maintain a regulatory system that can lead to the outcomes and expectations of its results." A management control system "is a set of internal information systems, simplifying the roots and facilitating information processing to help managers achieve an organization's goal on a continuous basis."

Operating systems are mandatory when managers and employees are not clear about what they expect; They have a good view of what they expected, but they did not attract the show; Or where, despite inspiration and inspiration for performance, managers and / or employees fail to maintain. According to Norbert Weiner, "Cybernetics studies the control and information theory in any machine or animal." The basic regulatory process based on the communication process involves the following steps: control, standardizing, evaluating performance, match performance on standards, rewarding good performance and / or taking appropriate measures and adjusting standards and actions when necessary.

Peter F. in 1954 The purpose of the Drunker is to perform objectives (MBO), a specific application of the cybernetic process of management control. In the MBO, goals / goals (specific, scalable, achieveable, realistic, and time-specific) are set up jointly and supervised. The MBO process involves six stages: developing overall company targets in key results; Establish specific targets for sub-units and individuals; Develop operational plans when identifying possible problem areas; Run self-direction and self-control between managers and colleagues; Review plans; Establishing reasonable performance when sufficient autonomy to implement plans. Some of the MBO restrictions are derived from lack of selfdirection and self-control, lack of performance guidelines and lack of observation to evaluate performance.

The Committee of Sponsoring Organizations (COSO) of the Treadway Commission (COSO) discussed the objectives of management controls under three sections: the effectiveness and efficiency of business operations; Credibility of financial statements; And applicable regulation and legal framework. An effective organization can achieve its purpose and objectives. The ability of the company to achieve results by using minimum resources. The financial report should have the characteristics of awareness, relevance and materialism and in accordance with accepted computational principles. A compliance target (regulatory issues (legal and other applicable requirements) is a must have at least one regimen for the corporations and does not establish or complement higher moral standards.

Expert management controls are based on control materials, time of execution of regulatory control and controls. Depending on the control object, management controls are grouped into action controls, results controls, and staff / cultural controls. Action controls are aimed at direct action at various levels of the organization. These can be further divided into behavioral rules, predictive actions and accountability. Focused on the consequences of actions taken rather than actions to take action on the results. Personnel / cultural controls influence people and institutional culture, and in the right culture the 
right people will eventually get the desired results, hoping to make the right actions.

Management controls can be categorized as formal controls and unauthorized controls based on the timing of regulatory enforcement, which can be classified into open loop controls and closed loop controls during control of the controls. Closed loop control mechanisms are categorized as further response (follow-up) control and feedfood (prior control) control. Different contextual factors influence the design and use of management control systems. These factors are: the nature and purpose of the organization; Organization structure and size; National culture; Corporate strategy and organizational variation; Competition strategy; Admin styles; Institutional slowdown Shareholders estimates and controls; And company life cycle. The nature and purpose of a company, which is a nonprofit or nonprofit organization, can have a major impact on management control systems. Profits, profits, and benefits of profits from non-profit organizations to non-profit organizations.

The organization structure creates an official model of job roles and responsibilities for individual employees and teams, and creates hierarchical construction and reporting relationships. The company's sizes affect the nature of the controls, such as rules, information papers, special character functions, and higher decentralization. The national culture of the country which operates the management control system of any organization affects. Geert Holstein has four facets of national cultures. Dimensions: power distance (hierarchical levels approved); Uncertainty over avoidance (preventing risk and confusion); Personalism / Collectivism (People's preference for working with people or team); And masculinity (competitive spirit, independent thinking, stiffness) / femininity (mutual, breeding nature). To achieve the goal between the company's ambitions and personal strategic business units, the management control system needs to have a good arrangement with corporate strategy. Management controls differ depending on diversification - relative or unrelated type. The selection of general competition strategy the overall cost affects leadership, difference, or vision - management control system.

Administrative styles (absolute or democratic, permission or directive) play an important role in influencing the behavior of employees in the organization and thus design and implement regulatory systems. Organizational slack indicates that capacity in an institution that is needed for general operations. It is created voluntarily or involuntarily and can be good or bad for the organization. Investors (investors, employees and managers, suppliers, customers, society, government etc.), individuals or groups of individuals influence the company's activities and activities. It is essential for corporations to see what the shareholders need when designing their management control systems.

Implementation of management control systems and governance may not work as a disadvantage for institutional goals. Administrators and employees are required to monitor the control system to see if they are encouraged to take action for the purpose of the organization, and thereby take corrective measures in designing and / or execution. Some exceptional consequences of management control systems are high quantitative and attempt to measure all possible steps, standard operating systems and information management that prevent innovation. Regulatory requirements are changing at any stage of the company's life cycle. Companies typically have five different phases of development and development such i as creativity, phase phase, decentralization phase, coordination phase and co-operation phase. Changing the rules of the organization's performance, control systems, and procedures, as well as changing the rules in an external environment, is a difficult process for a company to change from one step to another.

At creativity stage, decision maker is with employers and communication is informal. In the direction of the stage, the company receives a functional structure with revenue centers and cost centers; It implements accounting, budget and inventory management systems; There is formalization of communication and promotional schemes. At the decentralization phase, profit centers are created; The organizers are motivated by increased autonomy and incentives; Internal control and reporting systems help monitor the activities of low level managers. In coordination, companies receive divisional or production structure with investment centers; The proper systems for monitoring and control are in place; Strategic decisions are focused; And the promotions are associated with the company's performance. In the cooperative stage, a matrix structure was adopted; Teamwork, social controls, and self-discipline are often emphasized; Promotions are based on team performance; And focusing on innovation and 
problem solving. In addition to organizational increase, deterioration or limitations, a change in the control system used by a corporation can be altered or a completely new regulatory system is in effect.

\section{Conclusion}

The set of intrinsic communication structures that facilitate information processing for managers to assist the managers in the coordination of components and on a persistent basis is called management control system. The purpose of the proprietary control system is to coordinate the components of the organization and to carry out the steering of sections to achieve goals and goals for accomplishment of its overall purposes. The elements of a control system measure what is happening in the process. Comparing the expected function or standard performance determines the importance of what is happening; Performance expectations are low, and if the excelator has such cravings, influences change behavior; Detector and analyst and the intermediary communication between the intermediary and the communication communication network. Developing control systems in the organization is complicated and coordinated among many individuals, many functions, multiple time frames and many desires. Furthermore, controls are important because of strategic benefits to the business, facilitating "management through exemption", facilitating effective performance/ in business and providing self-solving mechanisms. Management control is more complicated because the management management system, systems, people and processes combine, rather than automated machines that control all inputs. There are many activities in the management control process, such as planning, coordination, consorting plans, obtaining information and processing and proper operation. Management control range includes strategic planning, management control and operational control. Strategic planning includes a road map that covers the longtime medium of business. Management control converts these plans into action points and informs them of the working group and reports outstanding results from the operation. Implementation of strategies implemented in the planning strategy will be implemented in planning management. The output of MCS is both financial and financial.

\section{References}

1) Management Control Systems (2nd Edition)

2) Anthony, R.N. Management control systems. Homewood, IL: Irwin; 1965

3) A Study of Management Control Systems (MCS) and School Performance by Devesh Baid

4) Effective Management Control, Theory and Practice Authors: Flamholtz, Eric G.

5) http://ycmou.digitaluniversity.ac/WebFiles/MBA FMG404_Management\%20Control\%20Systems.p df

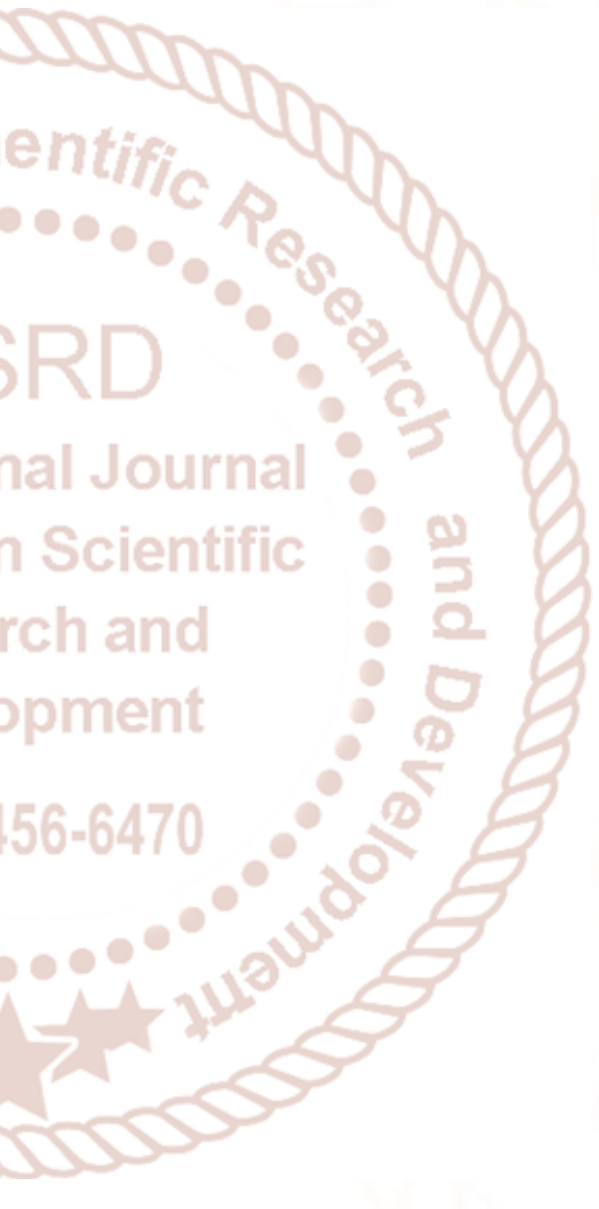

